Research Paper

\title{
Comparison of Floaters after Cataract Surgery with Different Viscoelastics
}

\author{
Jinsoo Kim, Hak Jun Lee, In Won Park, Soon Il Kwon ${ }^{\bowtie}$ \\ Department of Ophthalmology, Hallym University Sacred Heart Hospital, Anyang, Republic of Korea \\ $\triangle$ Corresponding author: Soon Il Kwon, Hallym University Sacred Heart Hospital, Anyang, Republic of Korea. Zip code: 14068; Phone: 82-10- 9075- 5854; Fax: \\ 82-31-380-3837; Email: magicham@gmail.com, magicham@hallym.or.kr \\ (C) Ivyspring International Publisher. This is an open access article distributed under the terms of the Creative Commons Attribution (CC BY-NC) license \\ (https://creativecommons.org/licenses/by-nc/4.0/). See http://ivyspring.com/terms for full terms and conditions.
}

Received: 2017.07.03; Accepted: 2017.09.01; Published: 2018.01.01

\begin{abstract}
Purpose: To investigate whether there is a difference between symptoms of floaters according to the type of ophthalmic viscosurgical devices(OVDs) used during phacoemulsification. Methods: A total of 112 eyes had undergone standard phacosurgery with the dispersive OVDs(Groupl). Group2 comprised 117 eyes that underwent phacosurgery with the dispersive OVDs, but between continuous curvilinear capsulorhexis and hydrodissection, some OVDs had been removed. Group3 included 120 eyes that had undergone phacosurgery with the cohesive OVDs. Results: 14 eyes (12.5\%) of Groupl had new-onset floater after surgery whereas 6 eyes $(5.13 \%)$ in Group2, and 7 eyes (5.83\%) in Group3 at the day after and a week after surgery. This was significantly higher in Groupl than Group2 and Group3, respectively $(p=0.047,0.049)$. Conclusion: Cataract surgery with dispersive OVD can predispose the eye to an increased floater symptom. Therefore, surgeons should consider release some OVDs during hydrodissection with dispersive viscoelastics and keep trying to avoid IOP surge during surgery.
\end{abstract}

Key words: Cataract surgery; Floaters; Ophthalmic viscosurgical devices; Phacoemulsification; Viscoelastics

\section{Introduction}

The surgical technique of phacoemulsification surgical technique has undergone significant improvement over the past several decades, and it results in improvement of visual acuity in many patients with cataract [1,2]. However, although the vision improves after phacoemulsification, many patients complain of discomfort. Floaters are one of the most common complaints in the ophthalmic care setting. Vitreous floaters usually occur with posterior vitreous detachment (PVD), and phacoemulsification can increase the risk of PVD. Typically, floaters are a sensation of gray or dark spots moving in the visual field and may persist for months or years [3-5]. Unlike the typical symptoms of floaters, some patients complain of tiny floaters that show up a day after cataract surgery and disappear within a few months. This usually happens with posterior capsular (PC) rupture, but it can also develop in a large number of patients who undergo phacosurgery without significant surgical complications. Another possible cause for this phenomenon is that lens particles and ophthalmic viscoelastic devices (OVDs) enter the vitreous cavity when the PC-anterior hyaloid membrane (AHM) barrier, which is made up of the zonule of Zinn and the AHM, ruptures [6]. Hydrodissection is considered the most likely cause of an AHM tear. Hydrodissection is the method of injecting fluid into the cortical layer of the capsule under the lens to separate the lens nucleus from the cortex capsule, and is an important step in the mobilization of the nucleus [7]. Since the intraocular pressure (IOP) becomes highest at this stage during phacosurgery, removing some OVDs before the hydrodissection could reduce the spike in IOP [8,9]. The aim of this study was to investigate whether there is a difference between symptoms of floaters according to the type of OVDs used during cataract surgery. In addition, we investigated if there is a difference in symptoms of floaters experienced after surgery when some OVDs are removed during hydrodissection. 


\section{Methods}

This retrospective observational study was conducted in accordance with the tenets of the Declaration of Helsinki and was approved by the Institutional Review Board of Hallym University Sacred Heart Hospital. The medical records of all patients who were older than 18 years and underwent phacoemulsification cataract surgery from March 2011 to June 2014 at the Hallym University Sacred Heart hospital, Korea, were reviewed. Exclusion criteria were patients who experienced adverse events during phacosurgery such as posterior capsular rupture, zonular dialysis, or radial tear. Difficult surgical cases such as brunescent cataract, shallow anterior chamber, zonular dehiscence, etc. were also excluded even though the surgery was not eventful. Patients with long axial length $(>27.0 \mathrm{~mm})$ or high myopia (spherical equivalent $>6.0 \mathrm{D}$ ), previous ocular surgery or trauma, and other ocular conditions which would be expected to be associated with an increased number of floaters, such as retinal tear, rhegmatogenous retinal detachment, diabetic retinopathy or asteroid hyalosis were excluded too.

We enrolled 349 eyes from 319 patients with cataracts, all patients had undergone uneventful standard phacoemulsification through a 3-mm incision with the implantation of an intraocular lens (IOL). A total of 112 eyes that had undergone standard phacosurgery with dispersive OVDs (DiscoVisc; Alcon) were assigned to Group 1. Group 2 comprised 117 eyes that had also undergone standard phacosurgery with dispersive OVDs, but between continuous curvilinear capsulorhexis (CCC) and hydrodissection, some OVDs had been removed through the incision site. Group 3 included 120 eyes that had undergone standard phacosurgery with cohesive OVDs (Microvisc; BohusBiotech); the OVDs had deliberately not been removed before hydrodissection. After cataract surgery, all patients underwent follow-up at 1 day, 1 week, 1 month, and 2 months. At every visit, participants were questioned about floater symptoms, and in case of presence of symptoms, how many floaters the participant could recognize, which is routine. Then, patients would have a non-mydriatic fundus photography, dilated fundus examination or optical coherence tomography if they were needed. The parameters of this study included demographics, preexisting eye diseases and details of cataract surgeries (including complications) undergone. In addition, best corrected visual acuity (BCVA) using the Snellen chart, IOP and the presence of floaters were evaluated. Intraocular pressure was measured with pneumatic tonometry.

Additionally, we evaluated the degree of floater symptoms. Every floater was assigned a score from 1 to 4 based on the number of new-onset floaters as follows: Score 1, no new-onset floater; Score 2, 1 or 2 new-onset floaters; Score 3, more than 2 and less than 10 new-onset floaters; Score 4, more than 10 new-onset floaters. We focused on patients with floaters with a score of 4 ; patients with scores of 1 through 3 were excluded as the new occurrence of a few floaters could be the manifestation of preexisting floaters that were not recognized under foggy vision.

Standard cataract surgery was performed by a single surgeon (S.K.). After a 3-mm corneal incision was made, the anterior chamber was filled with viscoelastic material (Disco Visc; Alcon). In the OVD removal group (Group 2), we removed some viscoelastics through the corneal incision site before hydrodissection. Then, hydrodissection was performed slowly and carefully with the use of a 10-ml disposable syringe equipped with a Healon needle through a side port. The needle was inserted beneath the anterior lens capsule. Next, phacoemulsification was performed on the lens nucleus and cortex as follows: The bottle height was adjusted to $78 \mathrm{~cm}$ above eye level, and then, the procedure was performed at a vacuum limit of 220 $\mathrm{mmHg}$ and a phacoemulsification power limit of $60 \%$. After filling up the capsular bag with viscoelastic material, we used an injector to implant an IOL (Tecnis 1-piece, Abbott medical optics) into the bag. After IOL implantation, the viscoelastic material was thoroughly washed via aspiration at a fixed flow rate of $25 \mathrm{ml} / \mathrm{min}$ and a maximum linear vacuum power of $500 \mathrm{mmHg}$. In Group 3, standard cataract surgery was performed with cohesive OVDs (Microvisc; BohusBiotech).

The chi-square test was applied for comparisons of initial demographic features such as age, sex, visual acuity, and IOP, and incidence of vitreous floater at follow-up visit between the groups 1, 2, and 3 . Analysis was performed using SPSS ver.12.0 (SPSS Inc., Chicago, IL, USA) and the level of significance was set at $p<0.05$.

\section{Results}

The Demographics of the study population are shown in Table 1. Mean age (Group 1: $67.53 \pm 13.55$ years, Group 2: $68.15 \pm 13.24$ years, Group 3: $66.89 \pm$ 13.75 years), sex ratio, and baseline IOP (Group1 : $13.15 \pm 3.14 \mathrm{mmHg}$, Group 2: $13.50 \pm 3.03 \mathrm{mmHg}$, Group 3: $13.170 \pm 3.23 \mathrm{mmHg}$ ) were not significantly different between the three groups (Table 1).

All 349 eyes underwent a standard 3-mm incision phacoemulsification without any significant complications. A high (30 $\mathrm{mmHg}$ or more) was observed in only 5 eyes ( 3 eyes in Group 1 and 2 eyes in Group 2) on the day after surgery, but normalized 
with medication within 1 week in all patients. No significant difference in postoperative IOP was observed among the three groups. Although there was no statistically significant difference in postoperative IOP between the three groups, new-onset floater symptoms after cataract surgery were experienced by 14 eyes in Group 1 (12.5\%), whereas only 6 eyes (5.13\%) in Group 2 and 7 eyes $(5.83 \%)$ in Group 3 experienced such symptoms on the day after surgery and a week after surgery, respectively, which was statistically different $(p=$ 0.047 and 0.049, respectively; Figure 1; Table 2, 3). Floater symptoms in Group 1 improved with time; therefore, no significant differences existed among three groups at 1 month and 2 months postoperatively.

Table 1. Demographics of study groups.

\begin{tabular}{llllll}
\hline & Group 1 & Group 2 & $p$-value & Group 3 & $p$-value \\
\hline Number of eyes & 112 & 117 & & 120 & \\
Age (years) & $67.53 \pm 13.55$ & $68.15 \pm$ & 0.613 & $66.89 \pm$ & 0.421 \\
& & 13.24 & & 13.75 & \\
Sex (Male : & $1: 0.81$ & $1: 0.85$ & 0.915 & $1: 0.91$ & 0.724
\end{tabular}

Female)

IOP $(\mathrm{mmHg})$

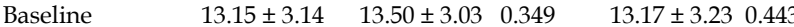

$\begin{array}{llllll}1 \text { day } & 16.64 \pm 4.90 & 16.70 \pm 5.01 & 0.913 & 16.01 \pm 4.01 & 0.935\end{array}$

1 week

$10.30 \pm 3.00 \quad 11.30 \pm 3.22 \quad 0.316 \quad 11.30 \pm 3.22 \quad 0.316$

Chi-square test $P<0.05$

Group 1: used dispersive ophthalmic viscosurgical devices (OVDs) and did not remove them before hydrodissection. Group 2: used dispersive OVDs and removed some dispersive OVDs before hydrodissection. Group 3: used cohesive OVD POD: postoperative day

Table 2. Comparison of number of patients with new-onset floater symptom between Groupl and Group 2.

\begin{tabular}{lllll}
\hline Patients & & Group 1 & Group 2 & $p$-valuet \\
\hline with & POD 1 day & $14(12.5 \%)$ & $6(5.13 \%)$ & 0.047 \\
new-onset & POD 1 week & $14(12.5 \%)$ & $6(5.13 \%)$ & 0.047 \\
floaters & $\begin{array}{l}\text { POD 1 } \\
\text { month }\end{array}$ & $10(8.93 \%)$ & $7(5.98 \%)$ & 0.395 \\
& $\begin{array}{l}\text { POD 2 } \\
\text { months }\end{array}$ & $8(7.14 \%)$ & $5(4.27 \%)$ & 0.348 \\
$\geq 10$ new-onset floaters & $6(5.35 \%)$ & 0 & 0.094 \\
during entire follow-up & & &
\end{tabular}

tMann Whitney U- test $P<0.05$

Group 1: used dispersive ophthalmic viscosurgical devices (OVDs) and did not remove them before hydrodissection. Group 2: used dispersive OVDs and removed some dispersive OVDs before hydrodissection. Group 3: used cohesive OVDs.

POD: postoperative day.

The floater scores show that 6 eyes in Group 1 $(5.35 \%)$ had more than 10 new floaters (score 4), whereas no eye in Group 2 and 1 eye in Group 3 had severe floater symptoms (Table 2, Table 3). Although Group 1 had more severe floater symptoms than Group 2 and 3, statistically significant differences did not exist in floater scores.

Table 3. Comparison of number of patients with new-onset floater symptom between Groupl and Group 3.

\begin{tabular}{|c|c|c|c|c|}
\hline \multicolumn{2}{|l|}{ Patients } & Group 1 & \multirow{2}{*}{$\begin{array}{l}\text { Group } 3 \\
7(5.83 \%)\end{array}$} & \multirow{2}{*}{$\begin{array}{l}p \text {-valuet } \\
0.049\end{array}$} \\
\hline With & POD 1day & $14(12.5 \%)$ & & \\
\hline new-onset & POD 1week & $14(12.5 \%)$ & $7(5.83 \%)$ & 0.049 \\
\hline floaters & POD 1month & $10(8.93 \%)$ & $10(8.33 \%)$ & 0.442 \\
\hline & $\begin{array}{l}\text { POD } \\
\text { 2months }\end{array}$ & $8(7.14 \%)$ & $10(8.33 \%)$ & 0.271 \\
\hline $\begin{array}{l}\geq 10 \text { new-on } \\
\text { during enti }\end{array}$ & $\begin{array}{l}\text { oaters } \\
\text { low-up }\end{array}$ & $6(5.35 \%)$ & $1(0.08 \%)$ & 0.125 \\
\hline \multicolumn{5}{|c|}{ tMann Whitney U- test $P<0.05$} \\
\hline \multicolumn{5}{|c|}{$\begin{array}{l}\text { Group } 1 \text { : used dispersive OVDs and did not remove dispersive OVDs before } \\
\text { hydrodissection. Group } 2: \text { used dispersive OVDs and removed some dispersive } \\
\text { OVDs before hydrodissection. Group } 3: \text { used cohesive OVDs. POD : postoperative } \\
\text { day. }\end{array}$} \\
\hline
\end{tabular}

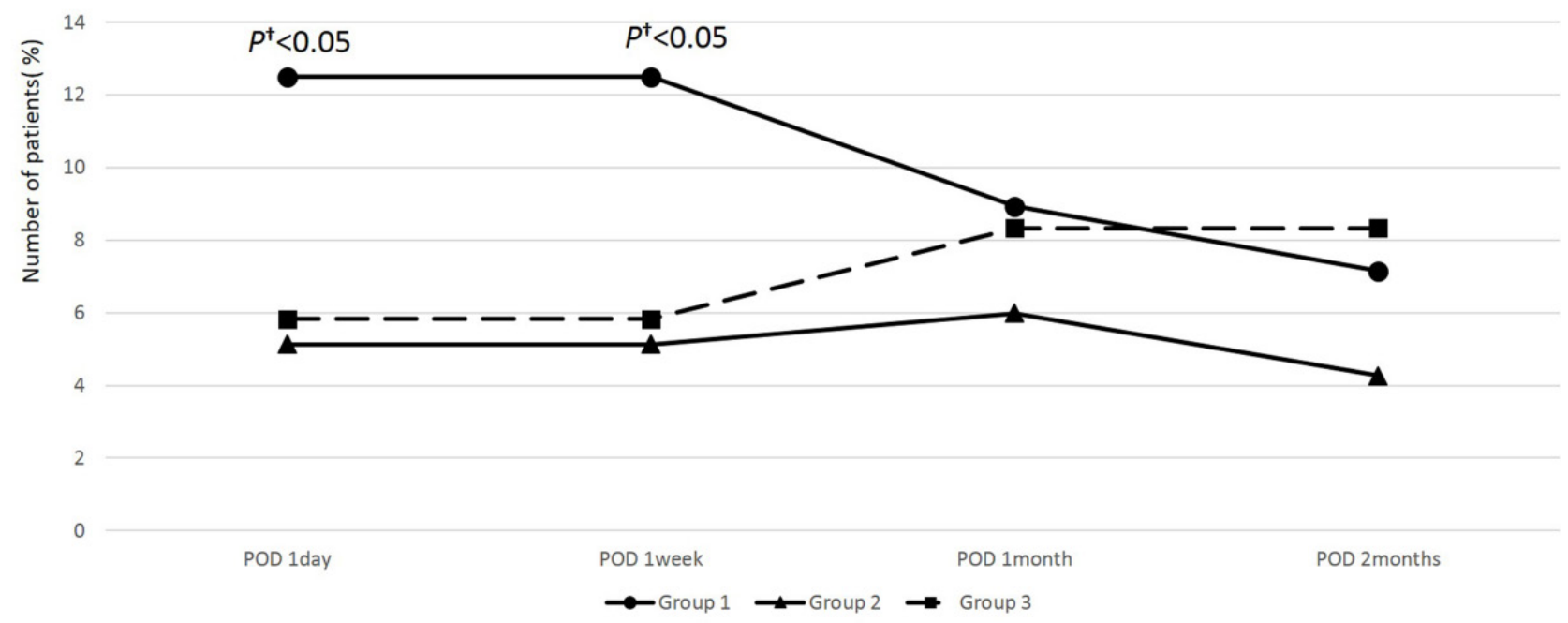

Figure 1. Percentage of patients with new-onset floater symptom during follow-up after cataract surgery in all study groups. The number of patients with postoperative floater symptom was significantly higher in Group 1 compared to other groups at 1 day and 1 week postoperatively. However, this difference was not significant after 1 month. ( + Mann Whitney $U$ - test < 0.05 ; Group 1: used dispersive ophthalmic viscosurgical devices (OVDs) and did not remove dispersive OVDs before hydrodissection. Group 2: used dispersive OVDs and removed some dispersive OVDs before hydrodissection. Group 3: used cohesive OVDs 


\section{Discussion}

A few months after we switched from cohesive to adhesive OVDs, which was newly added to our clinic, during cataract surgery, we observed that more patients complained of floaters after cataract surgery. Because the cataract surgeries were uneventful and nothing changed but newly added OVDs, we suspected new OVDs for these symptoms. Previous reports demonstrated that OVDs with higher molecular weight or sodium hyaluronate concentration might induce increased IOP and this changes in IOP stressed on PC-AMH barrier and could disrupt it $[10,11]$. We presumed that the IOP spike during surgery with the new OVDs was responsible for these symptoms, so we attempted to remove some OVDs during hydrodissection, since this surgical steps is the point at which the IOP is the highest. Since we checked postoperative floater symptoms routinely, we have compared the symptoms of floaters before and after the change of OVDs. PC-AHM barrier is thought to act as a mechanical barrier separating the physical and functional anterior portion of the eye from the posterior portion [10]. This barrier is well-maintained during normal phacoemulsification and aspiration operations; the anterior chamber material does not flow into the vitreous cavity. However, formation of an AHM tear offers a direct path from the anterior portion of the eye to the vitreous cavity.

Hydrodissection can be considered to be the most probable causative factor for AHM tear because the IOP becomes highest at this stage during phacoemulsification surgery. The AHM adheres firmly to the posterior lens capsule by means of the hyaloidocapsular Ligament of Weiger and a tear is frequently seen near the ligament $[12,13]$. In the presence of the injected viscoelastic materials, hydrodissection may place great stress on the Weiger ligament, causing a tear in the AHM. Viscoelastics normally play important roles in maintaining a surgical space in which CCC can be performed and also in protecting the corneal endothelium. Recently different properties of viscoelastics have been utilized for application in various situations, as the magnitude of increase in the IOP during hydrodissection varies widely depending on the type of viscoelastics used [14-16]. Dispersive OVDs have the ability to coat; they work well to keep the corneal endothelium protected during phacoemulsification. Meanwhile, cohesive OVDs are useful in creating and maintaining space in the anterior chamber and relatively easy to remove as a bolus. In the current study, because cohesive OVDs were spontaneously removed during the hydrodissection step, we did not have to deliberately remove them before hydrodissection. Because of these properties, cohesive OVDs are better than dispersive OVDs for avoiding IOP spikes, but less effective for coating and protecting the intraocular tissue. Ease of surgery, protection of the endothelium, and avoidance of an IOP spike are all factors that need to be considered while selecting an OVD; however, considering one factor might negatively affect the others. While using dispersive OVDs, it should always be kept in mind that careless maneuvers may lead to rupture of the PC-AHM barrier. Because unlike cohesive OVDs, it is difficult for dispersive OVDs to spontaneously pass through the corneal incision site during hydrodissection, it is necessary to avoid sealing the wound during hydrodissection.

In the current study, we used the side port for hydrodissection and the corneal incision was sealed when the IOP surged. If hydrodissection is performed through the main corneal wound, it could help avoid wound sealing, though not effectively.

We compared floater symptoms between different OVDs after cataract surgery because we assumed that because of its dispersive character, Discovisc might lead to a higher IOP spike than that caused by Microvisc, resulting in an AMH tear, which is the direct path between the anterior segment and the vitreous. Through this passage, tiny lens cortical materials or OVDs can spill over, resulting in floater symptoms, especially those with a score of 4 .

In our study, Group 1 patients showed more floater symptoms than those in Group 2 and Group 3 at 1 day and 1 week after the surgery. More eyes had a score 4 in Group 1 than in Group 2 or 3 [ 6 eyes (5.13\%) vs 0 or $1(0.08 \%)$, though the difference was not significant because of the small number of patients. These two results support our assumption.

Postoperative new-onset vitreous floaters have a tendency to disappear within a few weeks; there was no significant difference between the three groups at 1 month after surgery. I assume that, unlike floaters experienced prior to surgery, the postoperative floaters consisted of small lens particles or OVDs, which might be absorbed within weeks, and therefore the floater symptoms may be improve. Even though the floater symptoms might resolve in a month, the sequlae such as AHM tear or zonular damage remain for a long time and can result in delayed zonular dehiscence, subluxation or dislocation of the lens more than 10 years later.

Dispersive OVDs are not easily removed in the presence of high IOP, which can be useful in some circumstances, such as when a shallow anterior chamber is present due to high posterior pressure. In these cases, cohesive OVDs will easily become displaced through the wound site, therefore making it 
difficult to maintain the anterior chamber during phacoemulsification. Since dispersive OVDs can not only maintain anterior chamber in high IOP, but also protect corneal endothelium, they would be a better choice in these challenging cases.

The retrospective nature of the study and the small number of subjects were the limitations of this study; however, we could not enroll more subjects because of ethical constraints. We did not perform cataract surgery in Group 1 fashion since we noticed that patients in Group 1 complained of more floater symptoms than the patients in other groups. The subjectivity in quantifying floater symptoms is another limitation; because there is no objective method to evaluate floater symptoms, we had to rely on the patients' subjective views. To reduce such a subjective bias, we focused on severe floater symptoms only. Notwithstanding these limitations, to our best knowledge, this is the first study about postoperative floater symptoms, linking the characteristics of viscoelastic materials and IOP. This report contributes to our understanding of early onset postoperative floater symptom, to help reducing the occurrence of symptoms. Comparison of delayed complications such as lens subluxation or dislocation with this cohort will be a meaningful study in the future.

In conclusion, without release of the OVDs from the anterior chamber before hydrodissection, the eye has an increased risk of PC-AHM rupture during hydrodissection, especially with dispersive OVDs. The breakdown of the PC-AHM barrier potentially leads to numerous floaters. Thus, surgeons should consider releasing some OVDs during hydrodissection with dispersive viscoelastics and attempt to avoid IOP surge during surgery.

\section{Competing Interests}

All authors (Jinsoo Kim, Hak Jun Lee, In Won Park, Soon Il Kwon) declare that there are no conflicts of interest regarding the publication of this paper. The authors are responsible for the content and writing of the paper.

\section{References}

1. Davidson JA. Ultrasonic power reduction during phacoemulsification using adjunctive NeoSoniX technology. J Cataract Refract Surg. 2005; 31(5): 1015-9.

2. Soscoa W, Howard JG, Olson RJ. Microphacoemulsification with WhiteStar: a wound-temperature study. J Cataract Refract Surg. 2002; 28(6): 1044-6.

3. Hollands H, Johnson D, Brox AC, et al. Acute-Onset Floaters and Flashes Is This Patient at Risk for Retinal Detachment? JAMA. 2009; 302(20): 2243-9.

4. Hogan MJ. The vitreous, Its structure in relation to the ciliary body and retina. Invest Ophthalmol. 1963; 2: 418-45.

5. Schweitzer KD, Eneh AA, Hurst J, et al. Predicting retinal tears in posterior vitreous detachment. Can J Ophthalmol. 2011; 46(6): 481-5.

6. Thompson JT, Glaser BM. Role of lensectomy and posterior capsule in movement of tracer from vitreous to aqueous. Arch Ophthalmol. 1985; 103(3): 420-1

7. Faust KJ. Hydrodissection of soft nuclei. J Arn Intraocul Implant Soc. 1984; 10(1): 75-7.
8. Khng C, Packer $\mathrm{M}$, Fine $\mathrm{IH}$, et al. Intraocular pressure during phacoemulsification. J Cataract Refract Surg. 2006; 32(2): 301-8.

9. Oshika T, Okamoto F, Kaji Y, et al. Retention and removal of a new viscous dispersive ophthalmic viscosurgical device during cataract surgery in animal eyes. Br J Ophthalmol. 2006; 90(4): 485-7.

10. Kawasaki S, Suzuki T, Yamaguchi M, et al. Disruption of the posterior chamber - anterior hyaloid membrane during phacoemulsification and aspiration as revealed by contrast-enhanced magnative resonance. Arch Ophthalmol. 2009; 127(4): 465-70.

11. Kawasaki S, Tasaka Y, Suzuki T, et al. Influence of Elevated Intraocular Pressure on the Posterior Chamber-Anterior Hyaloid Membrane Barrier During Cataract Operations. Arch Ophthalmol . 2011; 129(6): 751-7.

12. Hogan MJ, Alvarado JA, Weddel JE. Histology of the Human Eye. Philadelphia: WB Saunders Company; 1974: 607-37.

13. Bron AJ, Tripathi RC, Tripathy BJ. Wolff's Anatomy of the Eye and Orbit, 8th ed. London: Chapman \& Hall; 1997: 443-53.

14. Modi SS, Davison JA, Walters T. Safety, efficacy and intraoperative characteristics of Discovisc and healon ophthalmic viscosurgical devices for cataract surgery. Clin Ophthalmol. 2011; 5: 1381-9.

15. Neumayer T, Prinz A, Findl O. Effect of a new cohesive ophthalmic viscosurgical device on corneal protection and intraocular pressure in small incision cataract surgery. J Cataract Refract Surg. 2008; 34(8): 1362-6.

16. Koch DD, Liu JF, Glasser DB, et al. A comparison of corneal endothelial changes after use of Healon or Viscoat during phacoemulsification. Am J Ophthalmol. 1983; 115(2): 188-201. 